Meta

Journal des traducteurs

Translators' Journal

\title{
Réflexions d'un traducteur technique généraliste
}

\section{Guy Lacoste}

Volume 21, numéro 1, mars 1976

La traduction et l'entreprise

URI : https://id.erudit.org/iderudit/003367ar

DOI : https://doi.org/10.7202/003367ar

Aller au sommaire du numéro

Éditeur(s)

Les Presses de l'Université de Montréal

ISSN

0026-0452 (imprimé)

1492-1421 (numérique)

Découvrir la revue

Citer cet article

Lacoste, G. (1976). Réflexions d'un traducteur technique généraliste. Meta,

21(1), 90-94. https://doi.org/10.7202/003367ar d'utilisation que vous pouvez consulter en ligne.

https://apropos.erudit.org/fr/usagers/politique-dutilisation/ 


\section{Réflexions d'un traducteur technique généraliste}

\section{Généralités}

N'ayant point fait de recherche sur la traduction technique au Québec, je me bornerai à parler du domaine dans lequel je travaille : la traduction technique générale dans une importante compagnie d'experts-conseils. La maison offre un éventail de services d'ingénierie dans des domaines très variés : énergie, transports, mines, métallurgic, pétrole et pétrolochimie, usines et environnement, pour ne nommer que ceux-là. Elle prend aussi en charge la réalisation complète d'unités de production « clés en main » ou «produit en main ». Dans une compagnie de ce genre, on attend donc du traducteur qu'il traduise des documents touchant un grand nombre de disciplines diverses et ce, dans les meilleurs délais.

\section{La spécialisation multidisciplinaire?}

$\mathrm{Si}$, comme l'écrivit le poète français Houdar de la Motte, «l'ennui naquit un jour de l'uniformité », on comprendra aisément que le traducteur technique qui passe sans transition d'un projet de cimenterie à une unité de matières plastiques, ou du balisage des pistes d'un aéroport à des études géologiques, n'a guère le loisir de s'ennuyer. Rassemblant toutes les connaissances éparses de physique, mécanique, chimie, mathématique, économique qu'il a emmagasinées pendant ses études, il doit les appliquer au travail de l'heure pour tâcher de "bien concevoir ce qui n'est pas toujours très clairement énoncé $»$. Pourtant, même si elle cause parfois des fatigues et de la tension, la diversité des disciplines n'en demeure pas moins un puissant facteur d'enrichissement des connaissances.

Alors que l'enseignement, la science et la technologie font de plus en plus appel à la spécialisation, le traducteur technique multidisciplinaire, lui, doit posséder un bagage de connaissances suffisamment vaste pour pouvoir traduire correctement dans de nombreuses disciplines. Est-ce à dire qu'il doit connaître à fond toutes ces disciplines et techniques? Assurément non! Il faut toutefois au traducteur une solide base multidisciplinaire, outre une bonne formation générale lui permettant de comprendre le texte de départ en posant des questions au spécialiste lorsqu'il bute sur une difficulté. 


\section{La collaboration technique}

Dans la pratique, il arrive malheureusement que des ingénieurs ou chefs de service aient, dans leur ignorance des techniques de la traduction, voire de la langue française, l'impression que le traducteur qui pose des questions ne connaît pas bien son métier. C'est une impression pénible qui frustre celui-ci. Certains veulent même avoir des explications sur ces questions, et cela peut entraîner des tensions et des pertes de temps dans l'exécution du travail. Parfois, par souci de rendement, ces personnes vont jusqu'à organiser le travail du traducteur. On peut ainsi faire traduire en français une nomenclature de pièces sans la planche de composition correspondante, et faire taper le texte par une dactylo anglophone unilingue. Bien que rares, de tels cas posent de réels problèmes. Il existe tout de même une majorité de personnes qui collaborent spontanément avec le traducteur, écoutent ses questions et ses suggestions et entretiennent avec lui des relations constructives.

La collaboration avec le spécialiste ou le «client» se révèle encore plus importante lorsque l'on se trouve en présence de textes de départ peu intelligibles et surchargés de répétitions. Il est alors indispensable de bien identifier les mots ou passages à éliminer pour parvenir à une rédaction claire et précise.

\section{Quel français utiliser?}

À ce sujet, je me permettrai de citer la « Norme du français écrit et parlé au Québec », Cahier de l'Office de la langue française, $n^{\circ} 1,1965$ :

C'est dans le seul domaine du lexique qu'il est possible d'accepter des divergences à la condition que les termes ainsi introduits soient construits selon la logique interne de la langue française, ne fassent pas double emploi avec des mots existants dont ils entraîneraient l'oubli à plus ou moins brève échéance, et servent à désigner des réalités nord-américaines pour lesquelles le français international n'a pas de termes appropriés.

Il faut éviter de considérer comme des canadianismes de bon aloi les mots dialectaux ou patois conservés dans la langue du peuple. De tels vocables, même s'ils sont encore vivants dans telle ou telle province dont sont originaires les Canadiens français, doivent être remplacés dans l'enseignement par les mots de la langue commune, le but de l'enseignement n'étant pas d'entériner ou de répandre tel mot patois, mais de faire apprendre le mot en usage dans le français du $\mathrm{Xx}^{\mathrm{e}}$ siècle.

Il faut aussi tenir compte de certains cas où la variante canadienne-française se justifie autant que le mot ou l'expression du français commun et peut même parfois leur être préférable. Exemples : fin de semaine, vivoir.

Bien que ces dernières années, la francisation de la langue technique ait fait d'énormes progrès, il n'en demeure pas moins que certains termes qui ne sont pas des canadianismes de bon aloi et ne se justifient pas autant que le mot ou l'expression du français commun sont imposés au traducteur à cause de la tradition et de l'ignorance de la terminologie internationale. Ainsi, le traducteur sera souvent obligé d'utiliser dans le contexte québécois «opérateur de machine-outil » et 《machiniste » au détriment de «conducteur de machine-outil », pourtant normalisé par le Code canadien des professions et le Bureau international du travail. 
Certaines personnes tiennent à tout prix à parler de "sablage » au lieu de "ponçage »; quant au «sablage », elles l'appelleront « décapage au sable ».

Cependant, comme les compagnies d'ingénieurs-conseils travaillent avec de nombreux pays étrangers francophones, y compris les pays où la langue de travail est le français, il est important, lorsqu'elles proposent des services à ces pays ou qu'elles y réalisent des travaux, d'utiliser les termes techniques du français commun et non leurs variantes canadiennes. Il ne faut pas oublier que cette terminologie technique commune est utilisée dans une trentaine de pays. D'ailleurs, plusieurs de ceux-ci comme l'Algérie, la Tunisie, le Maroc et le Zaïre font maintenant largement appel aux compagnies d'ingénierie canadiennes grâce auxquelles ils bénéficient de la technologie nord-américaine en langue française.

S'il importe de communiquer en français commun avec les pays francophones, il faut également leur fournir des documents (nomenclatures, dessins, schémas) comportant les abréviations françaises normalisées auxquelles ils sont habitués. Dans la famille des vis par exemple, lorsqu'on a «filister head $»$ ou «FH», il s'agit d'une «tête cylindrique bombée » ou «TCB », et « washer head » ou «WH » signifie «tête ronde à embase » ou «TRE ». Si l'on n'utilise pas ces abréviations, on en arrive à des longueurs incompatibles avec les impératifs de rendement. Au Canada cependant, il faut être très circonspect lorsque l'on introduit des abréviations. Tant que les entreprises québécoises ne travailleront pas réellement en français, il sera donc nécessaire d'écrire en toutes lettres certains termes que les autres francophones abrègent.

\section{Anglais britannique ou américain?}

Le Canada anglophone fait de grands efforts pour garder son individualité par rapport aux Etats-Unis. Pourtant, la terminologie de nos voisins du sud, se riant des frontières, pénètre à flots dans le monde de l'enseignement et du travail au Canada. Bien sûr, on reproduira encore longtemps des catalogues et autres ouvrages états-uniens en britannicisant «catalog » en « catalogue», en ajoutant un 《 $1 »$ à 《traveling » ou «enameling», et en insérant le 《u» traditionnel dans «neighbor», « honor » et « labor ». Inutile de se leurrer pourtant : la terminologie canadienne-anglaise est profondément américanisée. C'est, pour le traducteur technique, un facteur très important dont il doit tenir compte, faute de quoi il pourrait confondre «billion» en américain $\left(10^{9}\right)$ avec «billion» en anglais britannique $\left(10^{12}\right)$, ce qui pourrait provoquer une erreur d'un million de dollars!

\section{Organiser la traduction}

Je dois dire que plus les travaux sont importants, diversifiés et étendus dans le temps, plus le travail du traducteur technique sera complexe et plein d'aléas. En effet, lorsque plusieurs services parfois situés dans des provinces ou des pays différents travaillent en anglais pour un client étranger francophone, il est indispensable d'établir une collaboration entre les diverses personnes qui auront soit à traduire, soit à rédiger en français. Après consultations entre ces personnes, il y aura lieu de ficher tous les termes (deux fichiers, un avec entrées en français et l'autre, avec entrées en anglais) dans le but de constituer un lexique de première 
urgence qui sera distribué à tous les intéressés. Ce lexique servira à normaliser les termes utilisés dans les premières traductions du contrat qui risquent d'arriver très peu de temps après la signature de celui-ci. Par la suite, on profitera des temps morts pour élaborer des définitions dans les deux langues et les faire approuver par toutes les parties. Le lexique qui sera ainsi constitué, imprimé sur feuilles détachables, servira pendant toute la durée des travaux, s'enrichissant de nouveaux termes. Je sais par expérience que lorsqu'on connaît suffisamment un domaine et qu'on dispose de la terminologie bilingue correspondante, la précision et le rendement des traductions s'en trouvent fortement accrus. Il est également plus facile de sélectionner et de former d'autres traducteurs.

Nous avons vu plus haut que certaines personnes avaient du mal à accepter la terminologie du français commun. De ce côté-là, il y a cependant beaucoup de progrès. J'ai remarqué par exemple qu'une annonce parue dans la Presse du 18 octobre dernier indique que la Société d'énergie de la Baie James est le « maître d'ouvrage ». Je pense que les grands travaux réalisés en Afrique francophone ne sont pas étrangers à cette évolution. De toute façon, lorsqu'un terme français n'est pas accepté, il y a lieu de constituer un dossier avec, si possible, des exemples d'utilisation de ce terme au Canada. Il importe d'autre part d'être patient et d'accepter temporairement le terme local imposé. Lorsque le dossier devient irréfutable, il y a de fortes chances pour que le récalcitrant ne biffe plus le terme en litige.

\section{La documentation}

À mon avis, le traducteur devrait trouver dans la bibliothèque d'une entreprise à la pointe du progrès, les livres les plus récents dans tous les domaines. La réalité est pourtant tout autre. Si les livres en langue anglaise sont assez nombreux et variés, la documentation en français est d'une grande indigence. Pour peu, on croirait être dans une région anglophone. Sans compter que de nombreux livres en français datent de l'époque héroïque des grues à vapeur et des tramways à chevaux, à l'époque des vols interplanétaires et du transport aérien supersonique.

Je pense qu'avec l'adoption du SI, il n'y aura plus d'obstacle à la compréhension des ouvrages techniques français, et ce serait bien que de disposer d'une documentation de volume égal en français et en anglais. D'ailleurs, les ingénieurs pourraient eux aussi en profiter, en s'initiant au français technique. Mais, au-delà d'un certain nombre d'ouvrages, il serait souhaitable, tant pour le traducteur que pour les autres usagers, de disposer d'un système autre que les traditionnelles fiches en carton dans des tiroirs. En fait, l'idéal serait d'avoir un service de documentation informatisé, conçu de façon à pouvoir rechercher rapidement les documents et articles de revues du même domaine. Je sais qu'un tel système coûte cher, mais il peut aussi s'avérer très productif. De toute façon, une importante documentation qui ne peut être utilisée rapidement est soit inutile, soit cause de pertes de temps.

\section{La terminologie}

La terminologie est aussi vieille que le monde, mais peu de gens en avaient conscience. Maintenant, cette partie de la linguistique est enseignée à l'université qui forme des terminologues professionnels. 
C'est en effet de professionnels de la terminologie qu'ont besoin les grandes entreprises d'ingénierie. Si l'on considère l'énorme étendue et le degré de spécialisation du lexique avec tous les cas de polysémie qu'il comporte, on comprend l'importance d'un fichage organisé, tenant compte de la source du terme dans les deux langues, du niveau de langue, des particularismes linguistiques ainsi que de la technique ou science, avec ses spécialisations plus ou moins poussées.

Ainsi pour «bulldozer», dans le contexte d'équipements de niveleuses automotrices, on aura en première rubrique :

\section{ENGINS DE TERRASSEMENT \\ NTVELEUSES \\ ÉQUIPEMENTS}

Dans ce cas, le «bulldozer » est le «bloc de poussée » adaptable à l'avant de la niveleuse. Par contre, si l'on a comme seule rubrique :

ENGINS DE TERRASSEMENT, "BULLDOZER " signifiera :

$$
\begin{array}{ll}
\text { Bouldozeur } & \text { (F) } \\
\text { Bouteur } & \text { (F) } \\
\text { Boutoir } & \text { (C) }
\end{array}
$$

Cet exemple met aussi en relief la nécessité de normalisation du vocabulaire, une foison de synonymes ne pouvant que semer la confusion.

Il serait souhaitable que les grandes compagnies qui étudient tous les domaines du monde du travail prennent aussi conscience des problèmes de la traduction technique et surtout du fait que ces problèmes devraient être discutés avec des traducteurs compétents. Les décisions ainsi prises auraient un effet favorable sur le rendement et la qualité des traductions.

Pour atteindre ce but, il faudra probablement écrire encore beaucoup d'articles. Mais il faudrait surtout en écrire dans des quotidiens et des hebdomadaires.

J'ai essayé de présenter les problèmes du traducteur technique multidisciplinaire tels qu'ils m'apparaissent dans mon propre environnement : impossibilité du dialogue avec certains ingénieurs ou chefs de service, secrétaire unilingue anglophone imposée pour frapper des textes en français, obscurité et prolixité de certains textes de départ en français et résistance à la terminologie technique en usage dans les pays francophones.

La collaboration avec chaque spécialiste ainsi qu'avec le responsable de l'ensemble, la modernisation de la documentation, l'engagement de terminologues et, bien sûr, le choix logique des dactylos pour la frappe des textes, constituent, je crois, la prochaine étape à atteindre sur la voie pavée d'embûches de la traduction multidisciplinaire.

Certains de mes collègues ne seront peut-être pas d'accord avec moi sur un ou plusieurs points. Si la plume leur démange tant soit peu, je suis prêt à accueillir leurs critiques et leurs suggestions en osant espérer que nous trouvions ensemble des solutions utiles à tous.

GUY LACOSTE 